\title{
Assessment of Emergency Nurses Response Toward Caring of Victims During Disasters
}

\author{
Fatma Ali Mouhamed ${ }^{1}$, Mona Aly Mohamed ${ }^{2} \&$ Eman Ahmed Ismail ${ }^{3}$. \\ 1. Teacher at Secondary Nursing School in El Qusia city, Egypt. \\ 2. Assistant Professor of Critical care and Emergency Nursing, Faculty of Nursing, Assiut University, Egypt. \\ 3. Assistant Professor of Anesthesia and Intensive care Unit, Faculty of Medicine, Assiut University, Egypt.
}

\begin{abstract}
Background: Emergency nurses play a vital role in the response phase of disaster including immediate and acute care offered to victims during disasters, such as conducting triage. Aim of the study: To assess emergency nurses response toward caring of victims during disasters. Subject and Method: Purposive sample of 200 nurses working in the emergency departments at Assiut University Hospital and The ministry of Health Hospitals within 6 months period. The tool of data collection was the disaster response questionnaire. Results: Finding of the study revealed that less than half of studied nurses $(45,5 \%)$ had a poor level of knowledge regarding the disaster, response, education and training. Conclusion: Emergency nurses had poor level of knowledge regarding disaster, response, education and training. There was a statistical significant difference between knowledge of studied nurses in both hospital nurses group with (P. value $=<0.001$ ). While the third of studied nurses had a diploma in nursing and had a poor level of knowledge regarding care of victims during disasters. Recommendations: In-service training and education related to disaster management is required for all nurses' especially emergency nurses and should be tailored to the local needs and their actual competences.
\end{abstract}

\section{Keywords: Disaster, Disaster Response \& Emergency Nurses.}

\section{Introduction}

A disaster is defined by the international strategy for disaster reduction and World Health Organization ISDR \& WHO, (2009) as "a serious disruption of the functioning of a community or a society causing widespread human, material, economic or environmental losses which exceed the ability of the affected community or society to cope using its own resources" (Susan \& Joy, 2016).

Disaster criteria: Several criteria have been proposed to define disasters in terms of their consequences, at least one of the following criteria has to be fulfilled: ten or more people reported killed, one hundred people reported affected, a call for international assistance, unpredictable, unfamiliar, threat, speed and urgency and declaration of a state of emergency (Josephine et al., 2014).

Classification and types of disaster: there are different ways to classify disasters. Disasters are often classified according to their cause, their speed of onset (sudden or slow) and whether or not they are due to "acts of nature" or "acts of humans" (Matthew et al., 2018).

Disaster management includes four stages; prevention (mitigation), preparedness, response and recovery. Nurses have unique skills for all aspect of the disaster; this includes assessment, priority setting, collaboration and addressing both preventive and acute care needs. Nurses hold a major role in providing healthcare management and assistance, and allocating care during the time of the disaster and to reduce the impact of it on the community (Alexander, 2018).

Emergency nurses play a vital role in the response phase of disaster including immediate and acute care offered to victims during disasters, such as conducting triage, first aid, trauma care and emergency surgical care. The catastrophic event would have a considerable impact on the emergency department (ED) which is considered to be at the front line of the hospital response. Emergency nurses play a vital role in executing the department disaster plan and providing treatment for disaster victims (Fuad \& Yiannis, 2017).

\section{Significance of the Study}

In Egypt, there are many potential disasters that health care professionals might encounter. These disasters including both natural and manmade disaster like severe accidents (Traffic accidents as motor car accidents and train accidents), For example, there were 14,710 accidents recorded in 2016 which resulted in 5,343 deaths, 18,646 injuries and 21,089 damaged vehicles. Also death rates on intergovernorate roads record a total of 4,381 in the same year including highway records 962 deaths also there were 62 train accidents related fatalities and 164 injuries according to the central agency for public mobilization and statistics issued which press release with the statistics of car and train accidents in the year 2016 (Capms, 2017). 
Assessing the level of knowledge for nurses working in the emergency department's regarding the disaster response would identify both areas of weakness and strengths in their emergency response toward the care of victims during disasters. Future educational programs could then be developed to overcome the weaknesses identified in the study. A workforce that is better prepared would be able to function more cohesively according to need depending on each other more on a crisis situations than in normal dayto-day operations (Miller \& Brockie, 2017).

\section{Aim of the study}

The present study aims to

Assess emergency nurses response toward caring of victims during disasters.

\section{Research question}

What are emergency nurses' level of knowledge and response toward caring of victims during disasters?

\section{Subject \& Method \\ Research design}

A descriptive research design was used to conduct this study.

\section{Setting}

This study carried out in the emergency departments at Assiut Main University Hospital in the following areas; general emergency department and trauma emergency department. Also, the setting included the emergency departments in the Ministry of Health Hospitals at, EL Shamla hospital, EL Eman Hospital in Assiut City, EL qusia central hospital, Manfalut central hospital and Dairout central hospital.

\section{Sample}

A purposive sample of this study includes nurses working in the emergency departments at Assiut Main University Hospitals and The ministry of Health Hospitals. The sample size was 200 nursing staff; there were 68 in Assiut Main university hospital; 51 nurses in the general emergency department, 17 nurses in the Trauma emergency department and 132 nurses in the emergency departments associated to the Ministry of Health Hospitals as the following: 47 nurses at EL Shamla hospital, 27 nurses at EL Eman Hospital, 28 nurses at EL qusia central hospital, 12 nurses at Manfalut central hospital and 18 nurses at Dairout central hospital.

\section{Inclusion criteria}

The study included all nurses who provide direct and indirect patient care of victims at their emergency departments and who have at least one year of work at the departments.

\section{Exclusion criteria}

The study excluded all newly graduated nurses who work for less than one year in the emergency departments.

\section{Tool of data collection}

Tool: One tool was used in this study and was designed by the researcher based on the literature review for data collection (EPIQ, Survey monkey, 2014)

\section{Tool: Disaster response questionnaire}

This tool was developed by the researchers after reviewing the related literature. This tool aimed to assess emergency nurses' response toward caring for victims during disasters. This tool consists of multiple choices and true or false Arabic questionnaire related to their roles in response toward the care of victims during disasters. The questionnaire consists of 61 questions which consist of four main parts as the following:

Part I: This part includes the demographic data of the study sample characteristics, such as; code, six, marital status, years of experience in the emergency departments, years of experience in the nursing field, emergency department area, qualifications and level of career.

Part II: Nurses level of knowledge at emergency about disaster data which includes (21) multiplechoice questions to assess emergency nurses knowledge regarding disaster as definition, types, causes, phases, complications, Organizations involved in disaster and potential disasters in the community.

Part III: Role of Emergency nurses in disaster response data which includes (29) multiple choice and true or false questions to assess emergency nurses knowledge regarding their role in response toward victim's disaster as; questions about Emergency response to victims disaster, assessment, triage, Communication, Emotional support, resuscitation, Isolation and goal of response to victims disaster.

Part IV: Education and training about disaster response data which includes (11) multiple choice and true or false questions to assess emergency nurses' disaster education and training available and/or perceived by emergency nurses, experiences concerned with response to victims' disaster and scientific research.

The scoring system of nurses knowledge assessment tool

For multiple-choice questions and true or false questions emergency Nurses' knowledge as good, fair and poor level was carried as follows: all items have three alternative answers. It includes 61 questions for emergency nurses. A score value of one was awarded 
to each correct answer and score value of zero was awarded for incorrect answer. Scores categorized as; -Those who obtained less than 30.5 degrees $(<50 \%)$ were considered having a poor knowledge level, while those who obtained from 30.5 to 36.6 degrees (50 to $60 \%$ ) were considered having a fair level of knowledge. In addition, those who obtained scores above 36.6 degrees $(>60 \%)$ were considered having a good level of knowledge.

Working definition: Nurses response toward victims disaster means nurses level of knowledge regarding the care of victims during the disaster.

\section{Method}

The study was conducted throughout three main phases, preparatory phase, implementation phase and evaluation phase.

\section{1-Perparatory phase}

1. Through which the data collection tool was prepared and tested.

2. After taken the official permission from the faculty of nursing to conduct the study then it was delivered to the hospital authorities an official approval letter was obtained from the directors of the emergency departments (the general emergency department and the trauma emergency department at Assiut University Hospitals), in addition to the emergency departments in The ministry of Health hospitals (EL Shamla hospital, EL Eman Hospital in Assiut City, EL qusia central hospital, Manfalut central hospital and Dairout central hospital).

- these letters include permission to carry out the study after explaining the purpose, contents and the nature of the study.

3. A review of the current and international related literature in various aspects of this study using books, periodicals, and magazines were done.

\section{A pilot study}

It was conducted on $10 \%$ of the studied nurses (20) to test the feasibility and applicability of the tools and to estimate the time needed to fill the questionnaire. Based on the results of the pilot study, the necessary modifications in the sheet were done. The studied subjects were included in the actual study sample.

5. Tool which used in this study was developed in the Arabic language by the researchers based on reviewing the literature and tested for validity and reliability.

6. The reliability test for the tool (disaster response questionnaire) by using Alpha Cornbach's test $(\mathrm{R}=0.679)$ and Content validity was (0.82).

7. The tool were tested for content validity of research by a panel of 5 experts (4) from faculty of nursing (Staff of Critical care and Emergency
Nursing 3 Professors, and 1 lecturer) and one from faculty of medicine (Assistant professor Anesthesia) who reviewed the questionnaire for clarity, relevancy, and understanding and correction was carried accordingly.

\section{Ethical considerations}

1- Written consent was obtained from health teams that agree to participate in the study, after explaining the nature and purpose of the study.

2- Health team was assured that the data of this research will not be reused without second permission.

3- Confidentiality and anonymity was assured.

4- Health team has the right to voluntary participate or refuse participation in the study without any rationale at any time.

5- The objective of the study was explained by the researcher orally to the nurses, additionally to the written explanations in the covering letter of the questionnaire.

\section{2- Implementation phase and evaluation phase}

Once permission was granted to proceed with the proposed study, the researcher initiated data collection. The implementation phase involved the following issues:

1- At the initial interview, the researcher introduced herself to initiate a line of communication, explain the nature and purpose of the study before answering the questions to gain their consent and cooperation.

2- Data collection was done by the researchers, the head nurse and nurses of the department who helped the researchers to accomplish this work under the researcher's control.

3- Data were collected from emergency nurses in emergency and trauma departments at Assiut Main University Hospitals and emergency departments at the ministry of Health hospitals. Data were collected in six months approximately from December 2018 to May 2019.

4- The questionnaire to assess emergency nurses knowledge regarding their response toward the victim's disasters used for data collection from nurses included in the study.

5- The researchers interviewed each nurse individually to fill out the questionnaire sheet while they are on duty during any shift.

6- The researcher interviewed the emergency nurses at their break time and their answers were recorded immediately.

7- The average time taken for completing the questionnaire for each nurse was around 20-30 minutes.

8- During data collection, emergency nurses had the right to participate or not in the study. 
9- The questionnaire sheet included; demographic data, and knowledge about the emergency nurses' disaster, the role of emergency nurses during disaster response also, data about education besides training of disaster response.

\section{Statistical analysis}

The data were tested for normality using the Anderson-Darling test and for homogeneity variances prior to further statistical analysis. Categorical variables were described by number and percent $(\mathrm{N}$, $\%$ ), where continuous variables described by the mean and standard deviation (Mean, SD). We compared continuous variables by t-test and Anova Test. A two-tailed $\mathbf{p}<\mathbf{0 . 0 5}$ was considered statistically significant. All analyses were performed with the IBM SPSS 20.0 software.

\section{Results}

Table (1): Demographic characteristics of the nurses included in the study according to the hospital groups $(\mathbf{n}=\mathbf{2 0 0})$.

\begin{tabular}{|c|c|c|c|c|c|c|c|}
\hline \multirow[t]{2}{*}{ Characteristics } & \multicolumn{2}{|c|}{$\begin{array}{c}\text { University } \\
\text { hospital }(n=68)\end{array}$} & \multicolumn{2}{|c|}{$\begin{array}{c}\text { The ministry of health } \\
\text { hospitals }(n=132)\end{array}$} & \multicolumn{2}{|c|}{$\begin{array}{c}\text { Total } \\
(\mathbf{n}=\mathbf{2 0 0})\end{array}$} & \multirow[t]{2}{*}{ P. value } \\
\hline & N. & $\%$ & $\mathbf{N}$. & $\%$ & $\mathbf{N}$ & $\%$ & \\
\hline \multicolumn{8}{|l|}{ Age group } \\
\hline$<30$ years & 36 & 52.9 & 69 & 52.3 & 105 & 52.5 & \multirow{3}{*}{0.428} \\
\hline $30-40$ years & 28 & 41.2 & 48 & 36.4 & 76 & 38.0 & \\
\hline$>40$ years & 4 & 5.9 & 15 & 11.4 & 19 & 9.5 & \\
\hline Mean \pm SD & \multicolumn{2}{|c|}{$31.84 \pm 5.2$} & \multicolumn{2}{|c|}{$32.53 \pm 6.24$} & \multicolumn{2}{|c|}{$32.3 \pm 5.9$} & 0.433 \\
\hline \multicolumn{8}{|l|}{ Sex } \\
\hline Male & 16 & 23.5 & 59 & 44.7 & 75 & 37.5 & \multirow{2}{*}{$0.003 * *$} \\
\hline Female & 52 & 76.5 & 73 & 55.3 & 125 & 62.5 & \\
\hline \multicolumn{8}{|l|}{ Marital status } \\
\hline Single & 11 & 16.2 & 31 & 23.5 & 42 & 21.0 & \multirow{2}{*}{0.229} \\
\hline Married & 57 & 83.8 & 101 & 76.5 & 158 & 79.0 & \\
\hline \multicolumn{8}{|l|}{ Current profession } \\
\hline Nurse & 23 & 33.8 & 46 & 34.8 & 69 & 34.5 & \multirow{3}{*}{0.620} \\
\hline Head nurse & 11 & 16.2 & 28 & 21.2 & 39 & 19.5 & \\
\hline Technicians nurse & 34 & 50.0 & 58 & 43.9 & 92 & 46.0 & \\
\hline \multicolumn{8}{|l|}{ Experience year } \\
\hline$<5$ years & 12 & 17.6 & 31 & 23.5 & 43 & 21.5 & \multirow{4}{*}{0.446} \\
\hline $5-<10$ years & 33 & 48.5 & 49 & 37.1 & 82 & 41.0 & \\
\hline $10-<15$ years & 10 & 14.7 & 20 & 15.2 & 30 & 15.0 & \\
\hline$>15$ years & 13 & 19.1 & 32 & 24.2 & 45 & 22.5 & \\
\hline Mean \pm SD & \multicolumn{2}{|c|}{$10.68 \pm 6.08$} & \multicolumn{2}{|c|}{$11.17 \pm 7.33$} & \multicolumn{2}{|c|}{$11 \pm 6.92$} & 0.636 \\
\hline \multicolumn{8}{|c|}{$\begin{array}{l}\text { Years of experience in } \\
\text { department: }\end{array}$} \\
\hline$<5$ years & 14 & 20.6 & 46 & 34.8 & 60 & 30.0 & \multirow{4}{*}{$0.038 *$} \\
\hline $5-<10$ years & 43 & 63.2 & 58 & 43.9 & 101 & 50.5 & \\
\hline $10-<15$ years & 8 & 11.8 & 14 & 10.6 & 22 & 11.0 & \\
\hline$>15$ years & 3 & 4.4 & 14 & 10.6 & 17 & 8.5 & \\
\hline Mean \pm SD & \multicolumn{2}{|c|}{$8.54 \pm 3.75$} & \multicolumn{2}{|c|}{$8.27 \pm 4.77$} & \multicolumn{2}{|c|}{$8.37 \pm 4.44$} & 0.683 \\
\hline \multicolumn{8}{|l|}{ Qualifications } \\
\hline Diploma & 23 & 33.8 & 47 & 35.6 & 70 & 35.0 & \multirow{3}{*}{0.582} \\
\hline Technical institute & 34 & 50.0 & 57 & 43.2 & 91 & 45.5 & \\
\hline Bachelor & 11 & 16.2 & 28 & 21.2 & 39 & 19.5 & \\
\hline
\end{tabular}

Chi-square test $\quad *$ statistically significant difference $(p<0.05)$.

**statistically significant difference $(p<0.0 .01)$.

Independent $T$ - test 


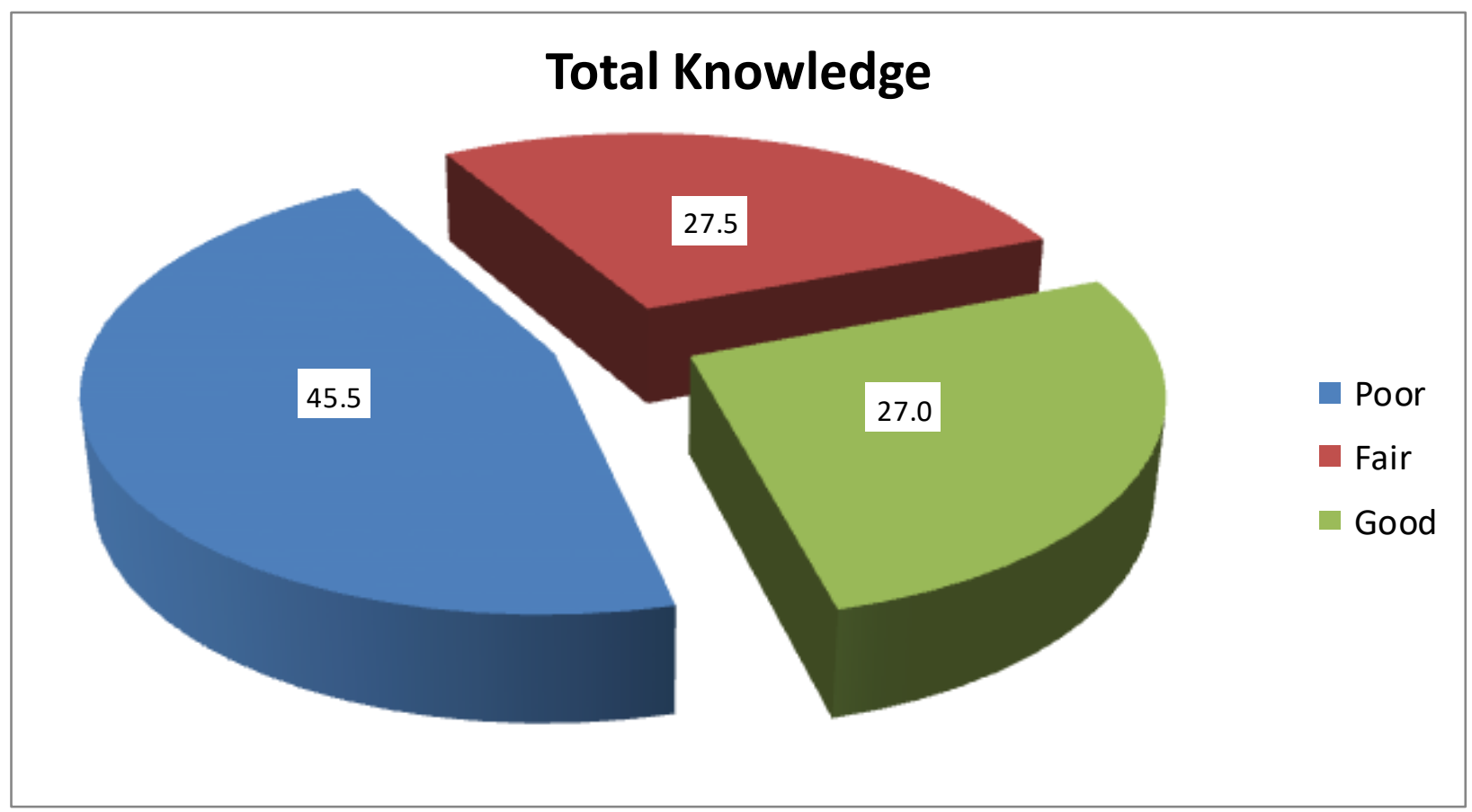

Figure (1): Percentage distribution of nurses according to their total level of knowledge.

Table (2): Nurses level of knowledge related to the disaster, disaster response, education and training according to hospitals groups $(\mathrm{n}=\mathbf{2 0 0})$.

\begin{tabular}{|l|c|c|c|c|}
\hline \multicolumn{1}{|c|}{ Knowledge } & Max score & $\begin{array}{c}\text { University hospital } \\
(\mathbf{n = 6 8})\end{array}$ & $\begin{array}{c}\text { The ministry of health } \\
\text { hospitals (n=132) }\end{array}$ & P. value \\
\hline Disaster knowledge & $\mathbf{2 1}$ & $12.65 \pm 3.32$ & $12.37 \pm 2.94$ & 0.548 \\
\hline Definitions & 2 & $0.84 \pm 0.54$ & $0.6 \pm 0.84$ & $0.033^{*}$ \\
\hline Types of disasters & 3 & $2.09 \pm 0.62$ & $1.99 \pm 0.53$ & 0.255 \\
\hline Causes of disasters & 6 & $4.51 \pm 1.1$ & $4.17 \pm 0.93$ & $0.019^{*}$ \\
\hline Phases of disaster & 3 & $1.01 \pm 0.98$ & $1.2 \pm 0.99$ & 0.199 \\
\hline Complications of disaster & 3 & $2.12 \pm 0.91$ & $2.28 \pm 0.81$ & 0.199 \\
\hline Organization involved in disaster & 1 & $0.44 \pm 0.5$ & $0.39 \pm 0.49$ & 0.522 \\
\hline Potential disasters in the community & 3 & $1.63 \pm 0.9$ & $1.73 \pm 0.85$ & 0.427 \\
\hline Disaster response knowledge & $\mathbf{2 9}$ & $14.51 \pm 4.64$ & $14.72 \pm 4.69$ & 0.769 \\
\hline $\begin{array}{l}\text { Emergency response to victims } \\
\text { disaster }\end{array}$ & 6 & $2.18 \pm 1.18$ & $1.94 \pm 1.46$ & 0.249 \\
\hline Assessment & 3 & $2.15 \pm 0.7$ & $2.19 \pm 0.71$ & 0.688 \\
\hline Triage & 4 & $1.6 \pm 0.98$ & $1.52 \pm 1.08$ & 0.575 \\
\hline Communication & 1 & $0.79 \pm 0.41$ & $0.81 \pm 0.39$ & 0.782 \\
\hline Aim of disaster response & 1 & $0.4 \pm 0.49$ & $0.48 \pm 0.5$ & 0.282 \\
\hline Emotional support & 2 & $1.16 \pm 0.84$ & $1.36 \pm 0.74$ & 0.084 \\
\hline Cardiopulmonary resuscitation & 3 & $1.41 \pm 0.87$ & $1.65 \pm 0.85$ & 0.062 \\
\hline $\begin{array}{l}\text { Nurses responsibility in response to } \\
\text { victims disasters }\end{array}$ & 4 & $2.09 \pm 1.34$ & $2.26 \pm 1.34$ & 0.398 \\
\hline $\begin{array}{l}\text { Types of support that would help } \\
\text { emergency nurses }\end{array}$ & 1 & $0.69 \pm 0.47$ & $0.7 \pm 0.46$ & 0.846 \\
\hline $\begin{array}{l}\text { Role of country in disaster } \\
\text { management }\end{array}$ & 1 & $0.9 \pm 0.31$ & $0.7 \pm 0.46$ & $0.002 * *$ \\
\hline Isolation & 3 & $1.15 \pm 1.25$ & $1.11 \pm 1.26$ & 0.827 \\
\hline
\end{tabular}




\begin{tabular}{|l|c|c|c|c|}
\hline \multicolumn{1}{|c|}{ Knowledge } & Max score & $\begin{array}{c}\text { University hospital } \\
(\mathbf{n = 6 8})\end{array}$ & $\begin{array}{c}\text { The ministry of health } \\
\text { hospitals (n=132) }\end{array}$ & P. value \\
\hline Training Courses & $\mathbf{1 1}$ & $5.16 \pm 2.28$ & $4.44 \pm 2.35$ & $0.039^{*}$ \\
\hline Training courses needed & 3 & $1.03 \pm 0.88$ & $0.69 \pm 0.8$ & $0.007 * *$ \\
\hline $\begin{array}{l}\text { Emergency nurses experience } \\
\text { concerned with response to disaster } \\
\text { victims }\end{array}$ & 6 & $3.03 \pm 1.45$ & $2.9 \pm 1.62$ & 0.585 \\
\hline Scientific research availability & 2 & $1.1 \pm 0.69$ & $0.85 \pm 0.73$ & $0.018^{*}$ \\
\hline Total Knowledge & $\mathbf{6 1}$ & $32.32 \pm 8.16$ & $31.53 \pm 8.24$ & 0.518 \\
\hline
\end{tabular}

Independent T-test * statistically significant difference $(p<0.05)$.

* *statistically significant difference $(p<0.01)$.

Table (3): Distribution of nurses' knowledge level according to hospitals groups $(\mathbf{n}=200)$.

\begin{tabular}{|c|c|c|c|c|c|c|}
\hline \multirow[t]{2}{*}{ Level of knowledge } & \multirow[t]{2}{*}{ Percentage } & \multicolumn{2}{|c|}{$\begin{array}{c}\text { University Hospital } \\
(\mathrm{n}=68)\end{array}$} & \multicolumn{2}{|c|}{$\begin{array}{c}\text { The ministry of health } \\
\text { Hospitals }(n=132)\end{array}$} & \multirow[t]{2}{*}{ P. value } \\
\hline & & N. & $\%$ & N. & $\%$ & \\
\hline$\overline{\text { Poor }}$ & $\overline{<50 \%}$ & 30 & $\overline{44.12}$ & 61 & 46.21 & \multirow{3}{*}{0.859} \\
\hline Fair & $50-60 \%$ & 18 & 26.47 & 37 & 28.03 & \\
\hline Good & $>60 \%$ & 20 & 29.41 & 34 & 25.76 & \\
\hline Mean \pm SD & & \multicolumn{2}{|c|}{$32.32 \pm 8.16$} & \multicolumn{2}{|c|}{$31.53 \pm 8.24$} & 0.518 \\
\hline
\end{tabular}

Chi-square test.

Table (4): Relationship between nurses knowledge scores with their demographic data according to hospitals groups $(\mathbf{n}=\mathbf{2 0 0})$.

\begin{tabular}{|c|c|c|c|c|}
\hline \multirow{2}{*}{ Characteristics } & \multicolumn{2}{|c|}{ University hospital(n=68) } & \multicolumn{2}{|c|}{ The ministry of health hospitals $(n=132)$} \\
\hline & N. & Mean \pm SD & N. & Mean \pm SD \\
\hline \multicolumn{5}{|l|}{ Age group } \\
\hline $20-<30$ years & 36 & $34.39 \pm 8.28$ & 69 & $34.04 \pm 6.91$ \\
\hline $30-<40$ years & 28 & $29.68 \pm 7.37$ & 48 & $28.85 \pm 8.91$ \\
\hline$>40$ years & 4 & $32.25 \pm 9$ & 15 & $28.53 \pm 8.45$ \\
\hline P. value & \multicolumn{2}{|c|}{0.070} & \multicolumn{2}{|c|}{$0.001 * *$} \\
\hline \multicolumn{5}{|l|}{ Sex } \\
\hline Male & 16 & $37.44 \pm 8.95$ & 59 & $33.49 \pm 7.66$ \\
\hline Female & 52 & $30.75 \pm 7.29$ & 73 & $29.95 \pm 8.4$ \\
\hline P. value & \multicolumn{2}{|c|}{$0.003 * *$} & \multicolumn{2}{|c|}{$0.013 *$} \\
\hline \multicolumn{5}{|l|}{ Marital Status } \\
\hline Single & 11 & $33.55 \pm 6.82$ & 31 & $32.29 \pm 6.79$ \\
\hline Married & 57 & $32.09 \pm 8.42$ & 101 & $31.3 \pm 8.65$ \\
\hline P. value & \multicolumn{2}{|c|}{0.591} & \multicolumn{2}{|c|}{0.559} \\
\hline \multicolumn{5}{|l|}{ Current profession } \\
\hline Nurse & 23 & $27.91 \pm 6.7$ & 46 & $26.37 \pm 7.86$ \\
\hline Head nurse & 11 & $34.82 \pm 8.1$ & 28 & $33.43 \pm 3.8$ \\
\hline Technicians nurse & 34 & $34.5 \pm 8.05$ & 58 & $34.71 \pm 8.18$ \\
\hline P. value & \multicolumn{2}{|c|}{$0.005 * *$} & \multicolumn{2}{|c|}{$<0.001 * * *$} \\
\hline \multicolumn{5}{|l|}{ Experience year } \\
\hline$<5$ years & 12 & $34 \pm 9.16$ & 31 & $32.81 \pm 5.23$ \\
\hline $5-<10$ years & 33 & $33.39 \pm 7.84$ & 49 & $34.73 \pm 7.45$ \\
\hline $10-<15$ years & 10 & $29.2 \pm 8.56$ & 20 & $27.6 \pm 10.6$ \\
\hline$>15$ years & 13 & $30.46 \pm 7.62$ & 32 & $27.84 \pm 7.97$ \\
\hline P. value & \multicolumn{2}{|c|}{0.365} & \multicolumn{2}{|c|}{$<0.001 * *$} \\
\hline
\end{tabular}




\begin{tabular}{|c|c|c|c|c|}
\hline \multirow{2}{*}{ Characteristics } & \multicolumn{2}{|c|}{ University hospital $(n=68)$} & \multicolumn{2}{|c|}{ The ministry of health hospitals $(n=132)$} \\
\hline & N. & Mean \pm SD & N. & Mean \pm SD \\
\hline \multicolumn{5}{|c|}{ Years of experience in the current emergency department: } \\
\hline$<5$ years & 14 & $33.36 \pm 8.76$ & 46 & $31.74 \pm 6.94$ \\
\hline $5-<10$ years & 43 & $33.07 \pm 7.52$ & 58 & $32.55 \pm 7.92$ \\
\hline $10-<15$ years & 8 & $26.88 \pm 9.08$ & 14 & $26.21 \pm 9.07$ \\
\hline$>15$ years & 3 & $31.33 \pm 10.79$ & 14 & $31.93 \pm 11.17$ \\
\hline P. value & \multicolumn{2}{|c|}{0.243} & \multicolumn{2}{|c|}{0.077} \\
\hline \multicolumn{5}{|l|}{ Qualifications } \\
\hline Diploma & 23 & $27.91 \pm 6.7$ & 47 & $26.72 \pm 8.21$ \\
\hline Technical institute & 34 & $34.5 \pm 8.05$ & 57 & $34.56 \pm 8.12$ \\
\hline Bachelor & 11 & $34.82 \pm 8.1$ & 28 & $33.43 \pm 3.8$ \\
\hline P. value & \multicolumn{2}{|c|}{$0.005 * *$} & \multicolumn{2}{|c|}{$<0.001 * *$} \\
\hline
\end{tabular}

Independent T- test ** statistically significant difference $(p<0.01)$. One way Anova* statistically significant difference $(p<0.05)$, ** statistically significant difference $(p<0.01)$

Table (5): Relationship between Nurses Knowledge Level with their demographic data according to hospitals groups $(n=200)$.

\begin{tabular}{|c|c|c|c|c|c|c|c|c|}
\hline \multirow{3}{*}{$\begin{array}{l}\text { Demographic } \\
\text { characteristics }\end{array}$} & \multicolumn{4}{|c|}{ University hospital(n=68) } & \multicolumn{4}{|c|}{$\begin{array}{l}\text { The ministry of health hospitals } \\
(n=132)\end{array}$} \\
\hline & $\begin{array}{c}\text { Poor } \\
(\mathbf{n}=30)\end{array}$ & $\begin{array}{c}\text { Fair } \\
(n=18)\end{array}$ & $\begin{array}{c}\text { Good } \\
(\mathbf{n}=\mathbf{2 0})\end{array}$ & \multirow[t]{2}{*}{ P. Value } & $\begin{array}{c}\begin{array}{c}\text { Poor } \\
(n=61)\end{array} \\
\end{array}$ & $\begin{array}{c}\text { Fair } \\
(\mathbf{n}=37)\end{array}$ & $\begin{array}{c}\text { Good } \\
(n=34)\end{array}$ & \multirow[t]{2}{*}{ P.value } \\
\hline & N. $(\%)$ & N. (\%) & N. $(\%)$ & & N. $(\%)$ & N. $(\%)$ & No. $(\%)$ & \\
\hline \multicolumn{9}{|l|}{ Age group } \\
\hline $20-<30$ years & $12(40)$ & $11(61.1)$ & $13(65)$ & \multirow{3}{*}{0.195} & $23(37.7)$ & $22(59.5)$ & $24(70.6)$ & \multirow{3}{*}{$0.017 *$} \\
\hline $30-<40$ years & $17(56.7)$ & $5(27.8)$ & $6(30)$ & & $27(44.3)$ & $13(35.1)$ & $8(23.5)$ & \\
\hline$>40$ years & $1(3.3)$ & $2(11.1)$ & $1(5)$ & & $11(18)$ & $2(5.4)$ & $2(5.9)$ & \\
\hline \multicolumn{9}{|l|}{ Sex } \\
\hline Male & $4(13.3)$ & $2(11.1)$ & $10(50)$ & \multirow{2}{*}{$0.004 * *$} & $24(39.3)$ & $15(40.5)$ & $20(58.8)$ & \multirow{2}{*}{0.156} \\
\hline Female & $26(86.7)$ & $16(88.9)$ & $10(50)$ & & $37(60.7)$ & $22(59.5)$ & $14(41.2)$ & \\
\hline \multicolumn{9}{|l|}{ Marital status } \\
\hline Single & $5(16.7)$ & $3(16.7)$ & $3(15)$ & \multirow{2}{*}{0.986} & $15(24.6)$ & $8(21.6)$ & $8(23.5)$ & \multirow{2}{*}{0.945} \\
\hline Married & $25(83.3)$ & $15(83.3)$ & $17(85)$ & & $46(75.4)$ & $29(78.4)$ & $26(76.5)$ & \\
\hline \multicolumn{9}{|l|}{$\begin{array}{l}\text { Current } \\
\text { profession }\end{array}$} \\
\hline Nurse & $15(50)$ & $6(33.3)$ & $2(10)$ & \multirow{3}{*}{$0.042 *$} & $35(57.4)$ & $8(21.6)$ & $3(8.8)$ & \multirow{3}{*}{$<0.001 * *$} \\
\hline Head nurse & $4(13.3)$ & $4(22.2)$ & $3(15)$ & & $6(9.8)$ & $15(40.5)$ & $7(20.6)$ & \\
\hline $\begin{array}{l}\text { Technicians } \\
\text { nurse }\end{array}$ & 11(36.7) & $8(44.4)$ & $15(75)$ & & $20(32.8)$ & $14(37.8)$ & $24(70.6)$ & \\
\hline \multicolumn{9}{|l|}{ Experience year } \\
\hline$<5$ years & $5(16.7)$ & $4(22.2)$ & $3(15)$ & \multirow{4}{*}{0.880} & $12(19.7)$ & $11(29.7)$ & $8(23.5)$ & \multirow{4}{*}{$0.002 * *$} \\
\hline $5-<10$ years & $13(43.3)$ & $8(44.4)$ & $12(60)$ & & $13(21.3)$ & $17(45.9)$ & $19(55.9)$ & \\
\hline $10-<15$ years & $6(20)$ & $2(11.1)$ & $2(10)$ & & $13(21.3)$ & $3(8.1)$ & $4(11.8)$ & \\
\hline$>15$ years & $6(20)$ & $4(22.2)$ & $3(15)$ & & $23(37.7)$ & $6(16.2)$ & $3(8.8)$ & \\
\hline \multicolumn{9}{|c|}{ Years of experience in department } \\
\hline $\begin{array}{lll}\begin{array}{l}\text { Less } \\
\text { years }\end{array} & \text { than } 5 \\
\end{array}$ & $6(20)$ & $5(27.8)$ & $3(15)$ & \multirow{4}{*}{0.814} & $20(32.8)$ & $15(40.5)$ & $11(32.4)$ & \multirow{4}{*}{0.248} \\
\hline $\begin{array}{ll}\text { from } & 5-10 \\
\text { years }\end{array}$ & $18(60)$ & $10(55.6)$ & $15(75)$ & & $23(37.7)$ & $18(48.6)$ & $17(50)$ & \\
\hline $\begin{array}{l}\text { from } 10-15 \\
\text { years }\end{array}$ & $5(16.7)$ & $2(11.1)$ & $1(5)$ & & $11(18)$ & $1(2.7)$ & $2(5.9)$ & \\
\hline $\begin{array}{l}\text { more than } 15 \\
\text { years }\end{array}$ & $1(3.3)$ & $1(5.6)$ & $1(5)$ & & $7(11.5)$ & $3(8.1)$ & $4(11.8)$ & \\
\hline
\end{tabular}




\begin{tabular}{|c|c|c|c|c|c|c|c|c|}
\hline \multirow{3}{*}{$\begin{array}{l}\text { Demographic } \\
\text { characteristics }\end{array}$} & \multicolumn{4}{|c|}{ University hospital(n=68) } & \multicolumn{4}{|c|}{$\begin{array}{l}\text { The ministry of health hospitals } \\
(n=132)\end{array}$} \\
\hline & $\begin{array}{c}\text { Poor } \\
(\mathbf{n}=30)\end{array}$ & $\begin{array}{c}\text { Fair } \\
(n=18)\end{array}$ & $\begin{array}{c}\text { Good } \\
(\mathbf{n}=\mathbf{2 0})\end{array}$ & \multirow[t]{2}{*}{ P. Value } & $\begin{array}{c}\text { Poor } \\
(n=61)\end{array}$ & $\begin{array}{c}\text { Fair } \\
(\mathbf{n}=37)\end{array}$ & $\begin{array}{c}\text { Good } \\
(\mathbf{n}=34)\end{array}$ & \multirow[t]{2}{*}{$P$.value } \\
\hline & N. $(\%)$ & N. $(\%)$ & N. $(\%)$ & & N. $(\%)$ & N. $(\%)$ & No. $(\%)$ & \\
\hline \multicolumn{9}{|l|}{ Qualifications } \\
\hline Diploma & $15(50)$ & $6(33.3)$ & $2(10)$ & \multirow{3}{*}{$0.042 *$} & $36(59)$ & $7(18.9)$ & $4(11.8)$ & \multirow{3}{*}{$<0.001 * *$} \\
\hline $\begin{array}{l}\text { Technical } \\
\text { institute }\end{array}$ & $11(36.7)$ & $8(44.4)$ & $15(75)$ & & $19(31.1)$ & $15(40.5)$ & $23(67.6)$ & \\
\hline Bachelor & $4(13.3)$ & $4(22.2)$ & $3(15)$ & & $6(9.8)$ & $15(40.5)$ & $7(20.6)$ & \\
\hline
\end{tabular}

Chi-square test $*$ statistically significant difference $(p<0.05) . * *$ statistically significant difference $(p<0.0 .01$

Table (6): Descriptive nurses' knowledge score regarding (disaster, disaster response, education and training) according to qualifications $(\mathrm{n}=\mathbf{2 0 0})$.

\begin{tabular}{|c|c|c|c|c|c|}
\hline Items of knowledge & $\begin{array}{l}\text { Max } \\
\text { score }\end{array}$ & $\operatorname{Diploma}(n=70)$ & $\begin{array}{c}\text { Technical } \\
\text { institute }(n=91)\end{array}$ & Bachelor $(n=39)$ & P. Value \\
\hline Disaster knowledge & 21 & $10.37 \pm 2.7$ & $13.41 \pm 2.79$ & $14.03 \pm 2.23$ & $<0.001 * *$ \\
\hline Definitions & 2 & $0.53 \pm 0.72$ & $0.8 \pm 0.79$ & $0.67 \pm 0.7$ & 0.073 \\
\hline Types of disasters & 3 & $1.77 \pm 0.68$ & $2.19 \pm 0.47$ & $2.1 \pm 0.31$ & 0.000 \\
\hline Causes of disasters & 6 & $3.79 \pm 0.96$ & $4.53 \pm 0.96$ & $4.62 \pm 0.81$ & $<0.001 * *$ \\
\hline Phases of disaster & 3 & $0.69 \pm 0.88$ & $1.27 \pm 0.92$ & $1.64 \pm 1.01$ & $<0.001 * *$ \\
\hline Complication of disaster & 3 & $2 \pm 0.88$ & $2.24 \pm 0.87$ & $2.59 \pm 0.55$ & $0.002 * *$ \\
\hline $\begin{array}{lll}\begin{array}{l}\text { Organization } \\
\text { disaster }\end{array} & \text { involved in } \\
\end{array}$ & 1 & $0.24 \pm 0.43$ & $0.43 \pm 0.5$ & $0.67 \pm 0.48$ & $<0.001 * *$ \\
\hline $\begin{array}{l}\text { Potential disasters in the } \\
\text { community }\end{array}$ & 3 & $1.36 \pm 0.96$ & $1.95 \pm 0.79$ & $1.74 \pm 0.59$ & $<0.001 * *$ \\
\hline $\begin{array}{ll}\text { Disaster } & \text { response } \\
\text { knowledge } & \\
\end{array}$ & 29 & $12.4 \pm 4.55$ & $16.01 \pm 4.67$ & $15.51 \pm 3.18$ & $<0.001 * *$ \\
\hline $\begin{array}{ll}\text { Emergency response } & \text { to } \\
\text { victims disaster } & \\
\end{array}$ & 6 & $1.97 \pm 1.2$ & $2.24 \pm 1.49$ & $1.59 \pm 1.31$ & $0.043^{*}$ \\
\hline Assessment & 3 & $1.87 \pm 0.72$ & $2.36 \pm 0.69$ & $2.28 \pm 0.51$ & $<0.001 * *$ \\
\hline Triage & 4 & $1.11 \pm 0.93$ & $1.77 \pm 1$ & $1.79 \pm 1.13$ & $<0.001 * *$ \\
\hline Communication & 1 & $0.8 \pm 0.4$ & $0.78 \pm 0.42$ & $0.87 \pm 0.34$ & 0.482 \\
\hline Aim of disaster response & 1 & $0.23 \pm 0.42$ & $0.6 \pm 0.49$ & $0.49 \pm 0.51$ & $<0.001 * *$ \\
\hline Emotional support & 2 & $0.93 \pm 0.79$ & $1.57 \pm 0.65$ & $1.31 \pm 0.8$ & $<0.001 * *$ \\
\hline $\begin{array}{l}\text { Cardiopulmonary } \\
\text { resuscitation }\end{array}$ & 3 & $1.23 \pm 0.75$ & $1.62 \pm 0.9$ & $2.08 \pm 0.66$ & $<0.001 * *$ \\
\hline $\begin{array}{l}\text { Nurses responsibility in } \\
\text { response to victims disasters }\end{array}$ & 4 & $1.57 \pm 1.27$ & $2.38 \pm 1.29$ & $2.9 \pm 1.1$ & $<0.001 * *$ \\
\hline $\begin{array}{l}\text { Type of support that would } \\
\text { help emergency nurses }\end{array}$ & 1 & $0.74 \pm 0.44$ & $0.62 \pm 0.49$ & $0.82 \pm 0.39$ & $0.040^{*}$ \\
\hline $\begin{array}{l}\text { Role of country in disaster } \\
\text { management }\end{array}$ & 1 & $0.73 \pm 0.45$ & $0.78 \pm 0.42$ & $0.82 \pm 0.39$ & 0.528 \\
\hline Isolation & 3 & $1.21 \pm 1.34$ & $1.29 \pm 1.2$ & $0.56 \pm 1.05$ & $0.007 * *$ \\
\hline Training courses & 11 & $4.34 \pm 2.58$ & $5.12 \pm 2.23$ & $4.28 \pm 2.05$ & 0.055 \\
\hline Training courses needed & 3 & $0.73 \pm 0.87$ & $0.86 \pm 0.8$ & $0.82 \pm 0.91$ & 0.628 \\
\hline $\begin{array}{l}\text { Experiences concerned with } \\
\text { response to disaster victims }\end{array}$ & 6 & $2.74 \pm 1.58$ & $3.24 \pm 1.64$ & $2.62 \pm 1.23$ & $0.045^{*}$ \\
\hline $\begin{array}{ll}\text { Scientific } & \text { research } \\
\text { availability } & \\
\end{array}$ & 2 & $0.87 \pm 0.74$ & $1.02 \pm 0.7$ & $0.85 \pm 0.74$ & 0.296 \\
\hline Total Knowledge & 61 & $27.11 \pm 7.72$ & $34.54 \pm 8.05$ & $33.82 \pm 5.29$ & $<0.001 * *$ \\
\hline
\end{tabular}

One way Anova* statistically significant difference ( $p<0.0 .05),{ }^{*}$ statistically significant difference $(p<0.0 .01)$ 
Table (7): Nurses knowledge level according to their qualifications.

\begin{tabular}{|c|c|c|c|c|c|c|c|c|}
\hline \multirow{3}{*}{$\begin{array}{c}\text { Total Knowledge } \\
\text { Level }\end{array}$} & \multirow{3}{*}{ Percentage } & \multicolumn{6}{|c|}{ Qualification } & \multirow{3}{*}{ P. value } \\
\hline & & \multicolumn{2}{|c|}{$\operatorname{Diploma}(n=70)$} & \multicolumn{2}{|c|}{$\begin{array}{c}\text { Technical } \\
\text { institute(n=91) }\end{array}$} & \multicolumn{2}{|c|}{$\operatorname{Bachelor}(n=39)$} & \\
\hline & & No & $\%$ & No & $\%$ & No & $\%$ & \\
\hline Poor & $<50 \%$ & 51 & 72.86 & 30 & 32.97 & 10 & 25.64 & \multirow{3}{*}{$<0.001^{* *}$} \\
\hline Fair & $50-60 \%$ & 13 & 18.57 & 23 & 25.27 & 19 & 48.72 & \\
\hline Good & $>60 \%$ & 6 & 8.57 & 38 & 41.76 & 10 & 25.64 & \\
\hline \multicolumn{2}{|c|}{ Mean \pm SD } & \multicolumn{2}{|c|}{$27.11 \pm 7.72$} & \multicolumn{2}{|c|}{$34.54 \pm 8.05$} & \multicolumn{2}{|c|}{$33.82 \pm 5.29$} & $<0.001 * *$ \\
\hline
\end{tabular}

Chi-square test $* *$ statistically significant difference $(p<0.0 .01$

One way Anova** statistically significant difference $(p<0.01)$

Table (1): Shows demographic characteristics of the studied nurses. It observed that the total number of nurses are (200) from different hospitals, (68) nurses from Assiut University Hospitals and (132) nurses from the Ministry of Health Hospitals. As regarding age, it observed that the majority of nurses $(52.3 \%)$ were between $20-<30$ years old in the ministry of health hospitals with a mean age of $32.53 \pm 6.24$ years old. As regarding sex, the majority of studied nurses $(55.3 \%)$ were female in the ministry of health hospitals. Regarding marital status, the majority of studied nurses $(76.5 \%)$ were married from the ministry of health hospitals. The study showed that the majority of studied nurses $(43.9 \%)$ were holding technical institute from the ministry of health hospitals, While years of experience in both nursing and the emergency departments, the study showed that $(37.1 \%)$ in years of experience in nursing with a mean years $11.17 \pm 7.33$ and $(43.9 \%)$ in years of experience in the emergency department at 5-<10 years with a mean years $8.27 \pm 4.77$. In addition, the table showed a statistical significant difference between the 2 hospitals groups related to sex with $\mathrm{P}$ value $=0.003$ and the majority of nurses were $(55.3 \%)$ and years of experience in the emergency department with $\mathrm{P}$. value $=0.038$ and the majority of nurses $(43.9 \%)$ were between 5 -to $<10$ years.

Table (2) \& Figure (1): Illustrated the percentage of studied nurses with poor knowledge level was $45.5 \%$, nurses with a fair level of knowledge were $27.5 \%$ and nurses with a good level of knowledge were $27 \%$.

NOTE: - Poor $<50 \%=<30.5$ degrees - Fair $50-60 \%$ $=30.5$ to 36.6 degrees $\quad-$ Good $>60 \%=>36.6$ degrees.

Table (3): This table showed that the total knowledge score regarding the disaster, disaster response, education and training of studied nurses were $\mathbf{6 1}$ with mean $\pm \mathrm{SD}(32.32 \pm 8.16)$ in University hospitals group and (31.53 \pm 8.24$)$ in The Ministry of Health hospitals group. While Knowledge about the disaster, disaster response, education and training were with mean \pm
SD (12.65 $\pm 3.32, \quad 14.51 \pm 4.64$ and $5.16 \pm 2.28)$ respectively in University Hospitals group and $(12.37 \pm 2.94,14.72 \pm 4.69$ and $4.44 \pm 2.35)$ respectively in the Ministry of Health Hospitals group.

There was statistical significant difference between knowledge of studied nurses in both groups related to the definition with $\mathrm{P}$. value $=0.033$. Regarding knowledge about causes of disasters with $\mathrm{P}$. value $=$ 0.019 , the role of the country in disaster management with $P$. value $=0.002$ and training courses with $P$. value $=0.007$ and scientific research with $P$. value $=$ 0.018 .

Table (4): Showed that (46.21\%) of studied nurses had a poor level of knowledge in the Ministry of Health hospitals group and (44.12) in University hospitals group regarding disaster, disaster response, education and training of the nurses.

Table (5): Showed that there was a statistical significant difference between knowledge of studied nurses in the ministry of health hospitals group and their age groups with $P$. value $=0.001$.

In addition, there was a statistical significant difference regarding knowledge of the studied nurses in both groups and their sex with P. value $=0.003$ in University hospitals, and with P. value $=0.013$ in the ministry of health hospitals group.

The table also, found that there was statistical significant difference of studied nurses knowledge and their profession with $P$. value $=0.005$ in University hospitals, and with P. value $=<0.001$ in the Ministry of Health hospitals groups.

The table added that; there was a statistical significant difference between knowledge of studied nurses in The Ministry of Health Hospitals group and their experience year in nursing with $\mathrm{P}$. value = $<0.001$.

There was a statistical significant difference regarding the level of knowledge of studied nurses and their qualifications with $\mathrm{P}$. value $=0.005$ in University hospitals group, and with $\mathrm{P}$. value $=$ $<0.001$ in the Ministry of Health Hospitals group. 
Table (6): Showed that there was a statistical significant difference regarding knowledge of studied nurses in the Ministry of Health hospitals group and their age group 30-<40 years old with $\mathrm{P}$. value = 0.017 as the majority of nurses $(44.3 \%)$ had poor knowledge level. The table also, revealed that; there was a statistical significant difference between studied nurses; knowledge in University Hospitals group and their sex with $\mathrm{P}$. value $=0.004$ also, the majority of nurses $(86.7 \%)$ were female and had a poor level of knowledge.

There was a statistical significant difference of knowledge of studied nurses between both hospital groups and their profession with $\mathrm{P}$. value $=0.042$ with the majority of nurses $(75 \%)$ were technical nurses in University hospitals group and had a good level of knowledge. While the Ministry of Health hospitals group with $\mathrm{P}$ value $=<0.001$ with the majority of nurses $(57.4 \%)$ holding a nursing diploma in the Ministry of Health hospitals group had a poor level of knowledge.

There was a statistical significant difference between knowledge of studied nurses in the Ministry of Health hospitals group and their experience years in nursing with $P$. value $=0.002$ while the majority of nurses $(37.7 \%)$ had a poor level of knowledge with the group of $>15$ years of experience in nursing.

There was a statistical significant difference between knowledge of studied nurses in both groups and their qualifications with $P$. value $=0.042$ while the majority of nurses (75\%) were technical nurses in University hospitals group and had a good level of knowledge, and the ministry of health hospitals group with $\mathrm{P}$ value $=<0.001$ and the majority of nurses (59\%) had nursing diploma with poor level of knowledge.

Table (7): Showed that nurses knowledge about the disaster, disaster response, education and training regarding their qualifications. From this table the maximum knowledge score of disaster, disaster response, education and training of the studied nurses were 61 and the mean \pm SD equal $27.11 \pm 7.72$ for nurses with nursing diploma, while 34.54 \pm 8.05 for nurses with technical institute and 33.82 \pm 5.29 for nurses had bachelor degree, The level of knowledge about disaster, disaster response, education and training were with mean \pm SD $(10.37 \pm 2.7,12.4 \pm 4.55$ and $4.34 \pm 2.58$ ) respectively and in nurses had nursing diploma, (13.41 $\pm 2.79, \quad 16.01 \pm 4.67$ and $5.12 \pm 2.23)$ respectively. Also, in nurses having technical institute with mean \pm SD were $(14.03 \pm 2.23$, $15.51 \pm 3.18$ and $4.28 \pm 2.05)$ respectively regarding nurses who had bachelor degree. There was a statistical significant difference regarding level of knowledge of studied nurses in both groups related to disaster knowledge with $\mathrm{P}$. value $=0.001$, disaster response knowledge with $\mathrm{P}$. value $=<0.001$ and total level of knowledge with P. value $=0.001$.

Table (8): showed that there was a statistical significant difference between knowledge of studied and their qualifications with P. value $=<0.001$ with the majority of nurses $(72.86 \%)$ holding a diploma in nursing and had a poor level of knowledge with mean $\pm \mathrm{SD}=27.11 \pm 7.72$.

\section{Discussion}

Disaster occurs suddenly, so having nurses with solid disaster knowledge will save more lives. According to the investigation and analysis of the knowledge of disaster rescue and training strategies of nurses. ((Tzeng et al., 2016). Therefore, the present study aimed to assess emergency nurses' response toward caring of victims during disasters.

The discussion will cover the main result findings as follow

Demographic characteristics of the studied nurses Based on the results of the present study, it was observed that the total number of nurses is (200) from different hospitals, (68) nurses from University hospital and (132) nurses from the Ministry of Health Hospitals, The majority of the nurses about half of them their age less than 30 years old, female, married and holding technical institute (diploma in nursing).

This was not in the same line with Ponikowski et al., (2016) who mentioned that; the administrators selected older age nurses to be able to perform tasks in the emergency units effectively.

As regarding years of experience in nursing and in emergency department it was observed that about half of them, their years of experience in nursing was around 5 to 10 years while the experience in the emergency department was around 5 to 10 years of experience.

As well, Shorofi \& Arbon, (2017) revealed in their study that the majority of nurses working in emergency departments their ages were ranged from 20-40 years old, married, female, and had a diploma of nursing and more than half of them, their experience was more than 5 years in the emergency department.

The result was incongruent with Baack \& Alfred, (2015) who reported that the majority of nurses who are working in ICU and emergency care had a bachelor degree in nursing.

As well, Emergency Nurses Association, (2017) stated that nurses with fewer years of experience may require maximum additional instructions before they are ready to take a patient assignment in one of the special units as nurses working in one clinical 
specialty may need further instructions to be acquired through the training program.

Regarding the nurses' knowledge about the disaster, disaster response, education and training The present study illustrated that majority of studied nurses had poor level of knowledge regarding disaster, disaster response, education and training.

Nurses' education experiences can enhance their positive knowledge on disaster management. The results of the survey on knowledge about disaster align with the findings of Alrazeeni, (2015) who mentioned that the integration of a course on disaster management in the emergency medical services (EMS) curriculum; along with practical training, will help to prepare students nurses in EMS to become better comprehend disaster management. Further, students of EMS asserted the needs of the integration of disaster management in the curriculum for the undergraduate program.

Similar findings were revealed in Sultan et al., (2017) study who reported that nurses' preparedness and trust regarding responses during disasters are influenced by their previous experiences, education, and training on disaster management. There was no disaster-planning program that had been approved by medical service centers at primary and hospital level, even though most of the nurses not received training about disaster management.

The findings were consistent with previous data from World Health Organization WHO, (2017); which reported that preparedness of nurses working in primary medical services is considered a low priority (WHO, 2017).

Further, it was reported that nurses did not fulfill most of their roles at the optimum level associated with the lack of preparation at all associated institutions.

The findings were supported by research in the Philippines by Labrague et al., (2016) \& Oztekin et al., (2016) who indicated that nurses were not well fully pre-pared to handle disasters because they did not understand disaster management protocol in their workplace.

Hutton et al., (2016) said that nurses who were working in the emergency unit needed additional education regarding disaster and disaster response. Grochtdreis et al., (2016) study showed clearly a knowledge gap among health professionals in understanding the concept of disaster and how to respond to specific disasters. Moreover, only a minority of the participants in Grochtdreis's study had received focused training on disaster preparedness and response. According to the respondents, the most valuable training topics would be communication skills, disaster $\mathrm{m}$ anagement, resource mobilization, health economics, and risk analysis.

Regarding the relationship between nurses knowledge level with their demographic data according to hospitals:

The result of the existing study showed that there was a statistical significant difference between knowledge of the studied nurses in the Ministry of Health hospitals group and their age group, sex, their profession, experience years in nursing and qualifications with $(\mathrm{P}$. value $=0.005)$ in University hospitals, and the Ministry of Health hospitals groups.

This findings not compatible with Gladston \& Nayak, (2017) who reported an opposite results of no association between age, marital status, education, and length of work on knowledge and perception of nurses preparedness in managing disasters. Levels of education and experience coupled with training on disaster response and preparedness showed significant differences in "skills" but did not have any effect on knowledge and evaluation in dealing with disasters. The findings of the research correspond to the results of the study.

Edmonson et al., (2017) stated that educational qualifications and experience of the training, and disaster management could improve disaster preparedness actions among nurses. Moreover, there was minimal evidence that education qualifications could improve cognitive ability related to emerging preparedness.

In addition, the present study revealed that there was a statistical significant difference between knowledge of studied nurses in the Ministry of Health hospitals group and their age group (30-<40 years old), technical degree of nursing education, and with a poor level of knowledge.

This agreed with Ben-Ishay et al., (2016) who found that participants with more than 10 years of clinical experience displayed greater self-reported scores in emergency response than nurses with less than 10 years of clinical experience. Similarly, a larger proportion of 946 nurses in mainland China with more clinical experience than without experience reported greater willingness to work in disaster relief efforts.

According to Alzahrani \& Yiannis (2017), who found the participants readiness for disaster responses were associated with nurses who had a bachelor's degree, emergency/intensive care experience, less than 10 years of nursing experience, and military background. These findings could help nurse educators to evaluate hospital nurses' readiness to respond to a disaster and recognize significant factors that require further 
training during undergraduate or continuing education.

Participants reported the highest disaster-readiness scores in clinical management, including physical assessment and equipment operation in an austere environment. However, the clinical experience of hospital nurses might not guarantee their effective performance in disaster conditions (Yin et al., 2015).

This study also, showed that nurses level of knowledge about disaster, disaster response, education and training was offered by qualifications, it is apparent that total knowledge score of disaster, disaster response, education and training of studied nurses were 61 with poor level of knowledge in nurses who had a nursing diploma, while good among nurses holding technical institute and bachelor degree.

In this regard, the data of the present research added that there was a statistical significant difference of knowledge among studied nurses with $\mathrm{P}$. value = $<0.001$ as more than half of the studied nurses had a diploma in nursing and had a poor level of knowledge. This finding the same line with Hammad, (2017) who found the level of education had an effect on the level of knowledge among nurses in his study entitled " The lived experience of nursing in the emergency department during a disaster" which revealed that the nurses' knowledge scores about disaster were poor among the institutional nurses than who holding bachelor degree. Also, he recommends developing particular clinical knowledge and skills through expose nurses to the educational program that addresses the disaster types and how nurses could provide disaster health care.

These results might be attributed to the fact that nurses working in the research and training hospitals had much more experience both in the profession and in the emergency care unit and almost all of them had participated in the in-service training programmes.

In this respect, Nehls et al., (2016) mentioned that nurses must be able to expand their knowledge in this area through ongoing education, journal, and seminars. Consequently, teaching programs for nursing staff constitute an important part.

These results agreed with Desseyn, (2017) who found that, approximately three-quarters of the nurses who participated in the program agreed that their knowledge about emergency preparedness and disaster response were improved.

Noguchi et al., (2016) stated that for professional nurses, continuing education is essential to safe and effective nursing care.

The emergency nurse plays a critical role in all disaster phases starting from the mitigation phase and continues throughout the disaster cycle, preparedness' response and recovery. Nurses are the frontline workers to provide effective care during disaster and crisis situations (Nakhaei et al., 2015). The researcher opinion; nurses need to have adequate knowledge and skills for an effective approach to respond to the critical situation especially in the disaster and catastrophic events. The amount of knowledge required to take care of victims during disaster cannot be obtained simply through experience on the unit or at the bedside (Natareno, 2018).

Finally, Nurses play a significant role in disaster preparedness, response, recovery and evaluation, especially in reducing vulnerability and minimizing risk in a disaster. Continuous training related to disaster management is required for all nurses, especially, emergency nurses. Training for disaster management simulation and distribution of nursing personnel in disaster areas must be considered with preparedness. Defining nurses' roles both in postdisaster preparedness and response must be taken into account with continuous training at various levels, including professional organization, governmental agencies, private organization, and the community.

\section{Conclusion}

The present study conducted that, emergency nurse's knowledge about disaster, disaster response, education and training at Assiut University hospital and the Ministry of Health hospitals over six months were poor among half of the nurses in both groups, emergency nurse's in the Ministry of Health hospitals group had a poor level of knowledge, while emergency nurse's with diploma in Assiut University hospitals group had a poor level of knowledge but the technical nurses had a good level of knowledge about the disaster, disaster response, education and training. Also there was a statistical significant difference of knowledge between the studied nurses in the both groups with $(\mathrm{P}$. value $=<0.001)$.

\section{Recommendations}

\section{The study recommended that}

1. In service training and education related to disaster response are required for all nurses' especially nurses working in the emergency department and training programs should be tailored according to the identified needs and their actual competences.

2. Furthermore interventional research can be carried out on interventional and qualitative studies focus on the same topic. 


\section{Reference}

1. Alexander D., (2018): Disaster and Emergency Planning for Preparedness, Response, and Recovery, natural hazard science, Oxford University.

2. Alrazeeni D., (2015): Saudi EMS students' perception of and attitudes toward their preparedness for disaster management. J Educ Pract., (6) P.P. 110:116

3. Alzahrani, F., \& Yiannis, K., (2017): Emergency nurse disaster preparedness during mass gatherings: a cross-sectional survey of emergency nurses' perceptions in hospitals in Mecca, Saudi Arabia. BMJ Open, 7(4), P.P.e013563.

4. Baack, S., \& Alfred, D., (2015): Nurses' preparedness and perceived competence in managing disasters. 4. Journal Of Nursing Scholarship, 45(3), 281-287

5. Ben-Ishay, O., Mitaritonno, M., Catena, F., Sartelli, M., Ansaloni, L., \& Kluger, Y., (2016): Mass casualty incidents-time to engage. World Journal of Emergency Surgery, 11(1), P.P. 8.

6. Capms (Central Agency for Public Mobilization and Statistics), (2017): Egypt country brief.

7. Desseyn, P., (2017): Triage is a process, not a destination. Journal of Emergency Nursing,3 (4), P.P.375:377.

8. Edmonson, C., McCarthy, C., Trent-Adams, S., McCain, C., \& Marshall, J., (2017): Emerging global health issues: a nurse's role. Online Journal of Issues in Nursing, 22(1), P.P. 2.

9. Emergency Nurses Association (ENA), (2017): Practice resource library. Retrieved May 28, 2017, from https://www.ena.org/practiceresources/resource-library/-incategory/ categories/ena/resources/practiceresources/emergency-preparedness.

10. Epiq (Emergency preparedness information questionnaire), (2014): Survey monkey web.http//WWW.surveymonkey.com

11. Fuad Al \& Yiannis K., (2017): Emergency nurse disaster preparedness during mass gatherings: a cross-sectional survey of emergency nurses' perceptions in hospitals in Mecca, Saudi Arabia, BMJ Open journal,7(4), P.P. 26:27.

12. Gladston S., \& Nayak R., (2017): Disaster preparedness among nurses working in a paediatric acute care setting of a tertiary hospital, south India. IOSR J Nurs Health Sci. (18), P.P. 25:35.
13. Grochtdreis, T., de Jong, N., Harenberg, N., Görres, S., \& Schröder-Bäck, P., (2016): Nurses' roles, knowledge and experience in national disaster pre-paredness and emergency response: A literature review. South Eastern European Journal of Public Health, 7(1), P.P. 34.

14. Hammad, K., (2017): The lived experience of nursing in the emergency department during a disaster. Flinders University online Library, 14(2), P.P. 87:94.

15. Hutton, A., Veenema, T., \& Gebbie, K., (2016): Review of the International Council of Nurses (ICN) framework of disaster nursing competencies. Prehospital and disaster medicine, 31(6), P.P. 680:683.

16. Josephine M., Michael H., Dennis P., Amy F., \& Amy H., (2014): The Role of Applied Epidemiology Methods in the Disaster Management Cycle, American journal of Public Health, 104(11): P.P. 2092: 2102.

17. Labrague L., Yboa B., \& McEnroe-Petitte D., (2016): Disaster preparedness in philippine nurses. J Nurs Scholarsh (48), P.P. 98:105.

18. Matthew F., Gabor D., Lauren M., Jamil D., Christina C., \& Scott L., (2018): Hospital Surge Capacity: A Web-Based Simulation Tool for Emergency Planners, Disaster Medicine and Public Health Preparedness, Society for Disaster Medicine and Public Health 12(4), P.P. 513:522.

19. Miller E., \& Brockie L., (2017): older adult's disaster lifecycle experience of the 2011 and 2013Queensland floods. International journal of disaster risk reduction, 22, P.P. 211: 218

20. Nakhaei, M., Khankeh, H., Masoumi, G., Hosseini, M., Parsa-Yekta, Z., Kurland, L., \& Castren, M., (2015): Impact of disaster on women in Iran and implication for emergency nurses volunteering to provide urgent humanitarian aid relief: A qualitative study. Australasian emergency nursing journal, 18(3), P.P. 165:172.

21. Natareno, K., (2018): Disaster Knowledge and Awareness of Nurses Related to Triage in Mass Casualty Incidents., conclusion chapter, P.P. 45

22. Nehls, N., Barber, G., \& Rice, E., (2016): Pathways to the PhD in nursing: an analysis of similarities and differences. Journal of Professional Nursing, 32(3), P.P. 163:172.

23. Noguchi, N., Inoue, S., Shimanoe, C., Shibayama, K., \& Shinchi, K., (2016): Factors associated with nursing activities in humanitarian aid and disaster relief. Plos One, 11(3), P.P. e0151170 
24. Oztekin S., Larson E., \& Akahoshi M., (2016): Japanese nurses' perception of their $€$ preparedness for disasters: quantitative survey research on one prefecture in Japan. Jpn J Nurs Sci. (13), P.P. 391:401

25. Ponikowski, P., Voors, A., Anker, S., Bueno, H., Cleland, J., Coats, A., \& Jessup, M., (2016): ESC Guidelines for the diagnosis and treatment of acute and chronic heart failure: The Task Force for the diagnosis and treatment of acute and chronic heart failure of the European Society of Cardiology (ESC). Developed with the special contribution of the Heart Failure Association (HFA) of the ESC. European journal of heart failure, 18(8), P.P. 891: 975.

26. Shorofi, S., \& Arbon, P., (2017): Complementary and alternative medicine (CAM) among Australian hospital-based nurses: knowledge, attitude, personal and professional use, reasons for use, CAM referrals, and sociodemographic predictors of CAM users. Complementary therapies in clinical practice, 27, P.P. 37:45.

27. Sultan M., Mary E., \& Al Grad M., (2017): Emergency Nurses Readiness for Disaster Response - An Explorative Study, American Research Journal of Nursing; 4(1), P.P. 1:10

28. Susan A., \& Joy K., (2016): Disaster Preparedness, Sultan Qaboos University Medical Journal, 16(1):P.P. e15:e19.

29. Tzeng W., Hsin P., Wei T., Chia- Huei L., Li C., \& Lu L., (2016): Readiness of hospital nurses for disaster responses in Taiwan: a cross sectional study. Nurse education today, 24(47). P.P. 134:140

30. WHO (World Health Organization), (2017): World Health Statistics 2017: Monitoring health for the SDGs. Retrieved November 2017, from http://www.who.int/ gho/ publications/ world_health_statistics/2017/ en

31. Yin Y., Turale, S., Stone, T., \& Petrini, M., (2015): Disaster nursing skills, knowledge and attitudes required in earthquake relief: implications for nursing education. Int. Nurs. Rev. 62 (3), P.P. 351:359. 